\title{
ECONOMIC CAPITAL ALLOCATIONS FOR NON-NEGATIVE PORTFOLIOS OF DEPENDENT RISKS
}

\author{
BY \\ EDWARD FURMAN ${ }^{1}$ AND ZiNOVIY LANDSMAN
}

\begin{abstract}
In this paper we explore the problem of economic capital allocations in the context of non-negative multivariate (insurance) risks possessing a dependence structure. We derive a general result and illustrate it with a number of useful examples. One such example, for instance, develops explicit expressions for the discussed economic capital decomposition rule when the underlying portfolio consists of dependent compound Poisson risks.
\end{abstract}

\section{KEYWORDS AND PHRASES}

Economic capital allocations, tail conditional expectation risk measure, multivariate non-negative dependent risks, multivariate Tweedie distributions, multivariate compound Poisson distributions.

\section{INTRODUCTION AND MOTIVATION}

In recent years, an increasing number of financial conglomerates have adopted economic (risk) capital frameworks. According to various authors (cf., e.g., Zaik et al., 1996) two central elements of such frameworks are: 1) holding sufficient capitals to cover risks, and 2) allocating economic capitals to each operating division or department.

At the international level, the immense importance of the aforementioned subjects can be clearly seen, on the one hand, in the European Commission's plans to apply the Basel II Bank Capital Adequacy Accord to all banks and investment firms in the European Union, and on the other, in the Commission's targets for the risk-based supervision of EU insurance companies, known as the Solvency II project.

At the national level, regulators around the world are increasingly applying the principles of the risk-based approach to all financial institutions under

1 Corresponding author, tel.:+1-416-736-2100 (Ext 33768), fax:+1-416-736-5757. 
their jurisdiction. In Israel, for instance, programs similar to the Basel II and Solvency II projects have been developed and are gradually being implemented.

Although the phenomena discussed above are becoming mandatory in nature in many countries, their practical utilization is far from straightforward. The complexity involved is in general explained by the following three main cornerstones of successful risk measurement (and therefore of the subsequent risk management):

1. The multivariate probabilistic model possessing a convenient dependence structure - to describe risks' behavior.

2. The choice of appropriate risk functional - to translate the implications of the model into risk parlance.

3. The (analytic) solutions for the latter in the framework of the former - to, actually, measure risk numerically.

In this paper we address all of these three issues. Namely, we consider portfolios of (insurance) risks following a multivariate dependent probabilistic model. The aforementioned class of distributions is referred to as the multivariate Tweedie family (MTwF), and it seems to answer well such peculiarities of the insurance industry demands as the non-negative support, unimodality, positive skewness, and tolerance for large risks. As special cases, MTwF contains e.g. the multivariate inverse Gaussian, multivariate gamma, multivariate stable, and multivariate compound Poisson distributions in the sense that their univariate margins are inverse Gaussian, gamma, positive stable, and compound Poisson, respectively. Furthermore, MTwF possesses a dependence structure, which is reflected in its covariance structure and allows for efficient modeling of multivariate portfolios of dependent risks (cf. Section 3 below for more details).

Relating to the second cornerstone above, we build the economic capital analysis developed herein on the popular tail conditional expectation (TCE) risk measure and the allocation rule based on it. Literally speaking, the former provides the necessary economic capital for the whole financial institution, whereas the latter resolves the problem of its subsequent allocation to various departments (operating divisions, sources).

Specifically, let us denote by $F$ and $\bar{F}=1-F$ the cumulative distribution function (cdf) and the decumulative distribution function (ddf), respectively, of a non-negative random variable (rv) $X$ representing the risk. The tail conditional expectation risk measure, which coincides with the expected shortfall (ES) and the conditional Value-at-Risk (CVaR) under the assumption of continuous distributions (cf., e.g., Hürlimann 2003; McNeil, Frei, Embrechts, 2005, Lemma 2.16), is then formulated as

$$
T C E_{q}[X]=\mathbf{E}\left[X \mid X>\operatorname{VaR}_{q}[X]\right]=\frac{1}{\bar{F}\left(\operatorname{VaR}_{q}[X]\right)} \int_{\operatorname{VaR}_{q}[X]}^{\infty} x d F(x),
$$


subject to $\bar{F}\left(\operatorname{VaR}_{q}[X]\right)>0, q \in(0,1)$ and

$$
\operatorname{VaR}_{q}[X]=\inf \{x: F(x) \geq q\} .
$$

Functional (1.1) possesses a number of appealing properties, which make it an attractive risk measure (cf. Artzner et al., 1999; Acerbi and Tasche, 2002; Tasche 2002). Very briefly speaking, TCE is sub-additive, scale- and translation-invariant, and satisfies first and second stochastic dominances (cf. Kaas et al., 2001; Denuit et al., 2005; Dhaene et al., 2006).

An alternative way to interpret equation (1.1) is to consider it a premium calculation principle (pcp), where the safety loading is determined by $\operatorname{VaR}_{q}[X]$ and is therefore proportional to the probability $q$. When one chooses this way of reasoning, one is in general interested in relatively small $q$ values, as distinct from the more common situations in the banking sector, when TCE denotes a risk measure, and thus $q$ is usually taken to be larger than 0.99 (cf. Furman and Landsman, 2006a). It should also be emphasized that TCE is a particular member of the class of distorted risk functionals and its generalization (cf. Denneberg, 1994; Wang, 1995, 1996; Wang et al., 1997; Hürlimann, 2006), as well as of the class of weighted pcp's (cf. Furman and Zitikis, 2008a). For statistical inferential results for the TCE risk measure, we refer to Brazauskas et al. (2008).

Another useful observation about TCE, made by Denault (2001) and Panjer and Jia (2001), implies that it can provide a natural decomposition of the total economic capital to its various constituents. Indeed, due to the additivity property of the expectation operator, one obtains that the 'risk contribution' of the $k$-th business line to the total risk $S=X_{1}+X_{2}+\cdots+X_{n}$ of the conglomerate is formulated as

$$
T C E_{q}\left[X_{k} \mid S\right]=\mathbf{E}\left[X_{k} \mid S>\operatorname{VaR}_{q}[S]\right]
$$

(cf. Hürlimann, 2004 for an alternative to 1.3; and Furman and Zitikis, 2008b for generalizations).

It should be noted that a significant number of risk measures other than TCE have been proposed, starting with the arguably oldest Value-at-Risk (cf. Leavens, 1945), and up to the distorted risk measures (cf. Denneberg, 1994; Wang, 1995, 1996; Wang et al., 1997). In addition, Furman and Landsman (2006b) proposed some tail variance-based risk measures, which on the one hand generalize equation (1.1), and on the other provide a tail-based counterpart to the classical variance and standard deviation pcp's. In general, the debate on which risk measure to apply in a given situation at hand is far from being over yet; however, this quite designing issue is far beyond the purposes of the herein discussion.

The main concerns of this paper are 1.) to suggest a new multivariate dependent family of distributions answering well such peculiarities of the insurance industry as non-negative support, unimodality and positive skewness, and 2.) to attempt evaluating (1.3) analytically, given this multivariate family. We note 
that Panjer and Jia (2001), Landsman and Valdez (2003), Cai and Li (2005) and Chiragiev and Landsman (2007) considered problems similar to our second point in the context of the multivariate normal, multivariate elliptical, multivariate phase type and multivariate Pareto distributions, correspondingly. Vernic (2006) evaluated equations (1.1) and (1.3) for multivariate skew-normal distributions, and Dhaene et al., (2008) have recently derived closed form approximations for the TCE based allocation for sums that involve lognormal risks. In the present paper, we evaluate (1.1) and (1.3) in the general context of the herein proposed multivariate Tweedie family, and we then illustrate our results by assuming the multivariate dependent compound Poisson distribution, which is an important particular case of MTwF.

We further proceed as follows: Section 2 discusses in detail some existing general results for non-negative independent risks. In spite of the restricting assumption of independence, these results allow for a valuable insight into the more interesting dependent problems. Then Section 3 introduces the multivariate Tweedie distributions and the particular case of interest in this paper: the multivariate compound Poisson family. Section 4 develops general expressions for equations (1.1) and (1.3) in the context of MTwF, and Section 5 illustrates the obtained results with some examples. Section 6 concludes the paper and discusses its main contributions.

\section{TCE AND THE ECONOMIC CAPITAL ALLOCATION FOR NON-NEGATIVE INDEPENDENT RISKS}

In this section we review some known results which are of interest. Unless otherwise stated, the results discussed here appear in Furman and Landsman (2005).

Let the non-negative rv $X$ have a finite expectation $\mathbf{E}[X]<\infty$. The TCE risk measure of $X$ turns out to be proportional to the expectation of $X$, as can be seen from the following note.

Note 2.1. Let $X^{*} \sim F_{X}^{*}$ denote the associated with $X r v$ with $c d f$

$$
F_{X^{*}}(x)=\frac{\mathbf{E}[X 1(X \leq x)]}{\mathbf{E}[X]}=\frac{1}{\mathbf{E}[X]} \int_{0}^{x} t d F(t),
$$

where $1(\mathcal{A})$ is the indicator function of the set $\mathcal{A}$. Then the tail conditional expectation risk measure of the risk $X$ can be formulated as

$$
T C E_{q}[X]=\mathbf{E}[X] \frac{\bar{F}_{X^{*}}\left(\operatorname{VaR}_{q}[X]\right)}{\bar{F}\left(\operatorname{VaR}_{q}[X]\right)} .
$$

Representation (2.1) seems to be not only attractive but also useful. For instance, noting that $X^{*}$ is stochastically greater than $X$, i.e., $P\left(X^{*}>x\right) \geq P(X>x)$ 
(cf., e.g., Patil and Rao, 1978), representation (2.1) immediately implies that $T C E_{q}[X] \geq \mathbf{E}[X]$, with equality only if $X^{*} \stackrel{d}{=} X$, where $\stackrel{d}{=}$ " stands for equality in distribution.

Another useful consequence of equation (2.1) is the appealing easiness of calculating TCE for such important rv's as gamma, Pareto, lognormal, Weibull, beta with continuous supports, and Poisson, binomial, negative binomial, logarithmic series, and hypergeometric with discrete supports. All these rv's are forminvariant with respect to their weighted counterparts (cf., e.g., Patil and Ord, 1976), and hence TCE straightforwardly follows using Tables 1 and 2 in loc. cite (cf. Furman and Zitikis, 2008a,c for a more detailed discussion of the usefulness of the associated $\operatorname{rv} X^{*}$ and its applications in actuarial science and finance). For the sake of completeness, we refer to Landsman and Valdez (2005) for an alternative representation of TCE in the class of exponential dispersion models (EDMs) that involves generalized hazard functions appropriately defined in this paper.

Let us further consider non-negative independent rv's $X_{j}$ possessing cdf's $F_{X_{j}}$. Also, denote the aggregate risk by $S=X_{1}+X_{2}+\cdots+X_{n} \backsim F$, and its associated counterpart by $S^{*}$. Clearly, TCE of $S$ is

$$
T C E_{q}[S]=\mathbf{E}[S] \frac{\bar{F}_{S^{*}}\left(\operatorname{VaR}_{q}[S]\right)}{\bar{F}\left(\operatorname{VaR}_{q}[S]\right)} .
$$

We are now in a position to consider the more general equation (1.3).

Lemma 2.1. The risk contribution of $X_{j}$ to $S$ is formulated in terms of TCE as

$$
T C E_{q}\left[X_{j} \mid S\right]=\mathbf{E}\left[X_{j}\right] \frac{\bar{F}_{S-X_{j}+X_{j}^{*}}\left(\operatorname{VaR}_{q}[S]\right)}{\bar{F}\left(\operatorname{VaR}_{q}[S]\right)},
$$

where $X_{j}^{*}$ is the independent associated counterpart of $X_{j}$.

In light of the latter expression, we are in general interested in the convolutions $S-X_{j}+X_{j}^{*}$. In some particular situations, these convolutions turn out to strongly simplify. Indeed, given some non-negative $\mathrm{rv} \xi_{j}$ independent on $X_{j}$, and if the associated counterpart of the latter rv can be rewritten as $X_{j}^{*}=X_{j}+\xi_{j}$, equation (2.3) reduces to

$$
T C E_{q}\left[X_{j} \mid S\right]=\mathbf{E}\left[X_{j}\right] \frac{\bar{F}_{S+\xi_{j}}\left(\operatorname{VaR}_{q}[S]\right)}{\bar{F}\left(\operatorname{VaR}_{q}[S]\right)} .
$$

To elucidate formula (2.4), we consider the two following examples.

Example 2.1. Let $X_{j}, j=1, \ldots, n$ be mutually independent $r v$ 's distributed gamma with the shape and rate parameters equal to $\gamma_{j}$ and $\alpha_{j}$, respectively, i.e., $X_{j} \sim$ 
$G a\left(\gamma_{j}, \alpha_{j}\right)$. In such a case, for $\gamma=\gamma_{1}+\gamma_{2}+\cdots+\gamma_{n}, \bar{\alpha}=\max \left(\alpha_{1}, \alpha_{2}, \ldots, \alpha_{n}\right)$ and setting without loss of generality $\alpha_{j}=\bar{\alpha}$, expression (2.3) can be rewritten as

$$
T C E_{q}\left[X_{j} \mid S\right]=\frac{\gamma_{j}}{\alpha_{j}} \frac{\bar{G}\left(\operatorname{VaR}_{q}[S] ; \gamma+K+1, \bar{\alpha}\right)}{\bar{G}\left(\operatorname{VaR}_{q}[S] ; \gamma+K, \bar{\alpha}\right)}
$$

where $\bar{G}(\because ; \gamma, \alpha)=1-G(\cdot ; \gamma, \alpha)$ denotes the ddf of some gamma distributed $r v$ with the shape and rate parameters equal to $\gamma$ and $\alpha$, respectively, and $K$ is $a$ specific discrete $r v$.

Formula (2.5) readily follows after noticing that in this case $X_{j}^{*} \sim G a\left(\gamma_{j}+1, \alpha_{j}\right)$, and therefore it can be represented by the convolution of two independent gamma $r v$ 's with common rate $\alpha_{j}$ and shapes equal to $\gamma_{j}$ and 1 . Therefore, representation (2.4) holds in this special case, where $\xi_{j} \sim G a\left(1, \alpha_{j}\right)$, and $S$ is the convolution of $n$ independent gamma rv's with shapes $\gamma_{j}$ and rates $\alpha_{j}$.

In our second example, equation (2.4) is satisfied again. Moreover, the $\mathrm{rv} \xi_{j}$ is equal to 1 over its whole domain, i.e., $P\left(\xi_{j}=1\right)=1$.

Example 2.2. Let $X_{j}, j=1, \ldots, n$ be mutually independent rv's distributed Poisson with $\lambda_{j}$, i.e., $X_{j} \backsim$ Poisson $\left(\lambda_{j}\right)$. Then it can be shown that $X_{j}^{*} \stackrel{d}{=} X_{j}+1$, and therefore, for $\lambda=\lambda_{1}+\lambda_{2}+\cdots+\lambda_{n}$, we have that

$$
T C E_{q}\left[X_{j} \mid S\right]=\lambda_{j} \frac{\overline{P o}\left(\operatorname{VaR}_{q}[S]-1 ; \lambda\right)}{\overline{P o}\left(\operatorname{VaR}_{q}[S] ; \lambda\right)},
$$

where $\overline{P o}(\because, \lambda)=1-\operatorname{Po}(\because ; \lambda)$ denotes the ddf of a Poisson rv parameterized by $\lambda$.

It can be then noted that the contribution of $X_{j}$ to $S$ is stipulated in this special case by the expectations $\mathbf{E}\left[X_{j}\right]=\lambda_{j}$ only. Consequently, and due to equation (2.1), the relative contribution is

$$
\frac{T C E_{q}\left[X_{j} \mid S\right]}{T C E_{q}[S]}=\frac{\mathbf{E}\left[X_{j}\right]}{\mathbf{E}[S]}
$$

which is surprisingly very simple. We note that (2.7) does not hold in general (cf., e.g., Example 2.1 above).

To conclude, it should be noted that, although $X_{1}, \ldots, X_{n}$ are independent, this is not the case for the pair $X_{j}$ and $S$ which is certainly dependent. In light of this, Note 2.1 and Lemma 2.1 may prove to be of high practical importance. Other valuable applications of the aforementioned results are demonstrated in Section 4 below. 
In the next section we depart from the assumption of independence, and we introduce the multivariate Tweedie family of distributions along with its member which is of particular interest in this paper, the multivariate compound Poisson distribution.

\section{Multivariate Tweedie Distributions}

Exponential dispersion models play a prominent role in statistics and actuarial science. This can be explained by the high level of generality that EDMs enable in the context of statistical inference for such widely popular distribution functions as normal, gamma, inverse Gaussian, stable, and many others. The specificity characterizing statistical modeling of actuarial subjects is that the underlying distributions mostly have non-negative supports and many EDMs possess this important phenomenon.

Although univariate EDMs are considerably rich and widely applied, in the multivariate context the case is very different. Unfortunately, the so-called natural multivariate EDMs are not as rich as the univariate ones. Namely, they do not include important multivariate distributions whose univariate margins are, say, inverse Gaussian, gamma or compound Poisson. Moreover, the only valuable continuous member of such a natural multivariate extension of the univariate EDMs is the multivariate normal distribution (cf. Bildicar and Patil, 1968 for more details).

To overcome the aforementioned penury, we suggest a new multivariate Tweedie $^{2}$ family of distributions. We use an extended version of the bivariate reduction method of Mardia (1970), which is further formulated in a quite general form and referred to as the multivariate reduction technique (cf., e.g., Joe, 1997; Hürlimann, 2007a; Furman, 2008; and references therein).

To briefly follow our main reasoning, let $\mathbf{Y}=\left(Y_{0}, Y_{1}, \ldots, Y_{n}\right)^{T}$ be a random vector consisting of $(n+1)$ mutually independent additive Tweedie rv's, i.e., $Y_{i} \sim T w_{p}\left(\theta_{i}, \lambda_{i}\right), i=0,1, \ldots, n$, possessing, under certain conditions, probability distribution functions (pdf's) or the probability mass functions (pmf's) of the form

$$
f(x)=h(x ; \lambda) \exp \left(\theta x-\lambda \kappa_{p}(\theta)\right),
$$

where, for $\alpha=(p-2) /(p-1)$,

$$
\kappa_{p}(\theta)=\left\{\begin{array}{ll}
e^{\theta} & p=1 \\
-\log (-\theta) & p=2 \\
\frac{(\alpha-1)}{\alpha}\left(\frac{\theta}{(\alpha-1)}\right)^{\alpha}, & p \neq 1,2
\end{array} .\right.
$$

and $\theta \in \Theta_{p}$ such that the domain $\Theta_{p}$ is the largest interval for which $\kappa_{p}(\theta)$ is finite (cf. Jorgensen, 1997 for details).

2 EDMs are classified by their unit variance functions $V(\cdot)$. EDMs having power form variance function $V(\mu)=\mu^{p}, p \in(-\infty, 0] \cup[1, \infty)$ are called Tweedie EDMs (cf., e.g., Jorgensen, 1997). 
Also, let

$$
A=\left(\begin{array}{cccccc}
\frac{\theta_{0}}{\theta_{1}} & 1 & 0 & 0 & \ldots & 0 \\
\frac{\theta_{0}}{\theta_{2}} & 0 & 1 & 0 & \ldots & 0 \\
\frac{\theta_{0}}{\theta_{3}} & 0 & 0 & 1 & \ldots & 0 \\
\vdots & \vdots & \vdots & \vdots & \ddots & \vdots \\
\frac{\theta_{0}}{\theta_{n}} & 0 & 0 & 0 & \ldots & 1
\end{array}\right)
$$

Theorem 3.1. Let $\mathbf{X}=A \mathbf{Y}$. Then the $j$-th univariate marginal distribution of $\mathbf{X}$ is

$$
\frac{\theta_{0}}{\theta_{j}} Y_{0}+Y_{j}=X_{j} \backsim\left\{\begin{array}{ll}
T w_{1}\left(\theta, \lambda_{0}+\lambda_{j}\right), & p=1 \\
T w_{2}\left(\theta_{j}, \lambda_{0}+\lambda_{j}\right), & p=2 \\
T w_{p}\left(\theta_{j}, \lambda_{0}\left(\frac{\theta_{0}}{\theta_{j}}\right)^{\alpha}+\lambda_{j}\right), & p \neq 1,2
\end{array} .\right.
$$

where $\alpha=(p-2) /(p-1)$.

The theorem ensures that MTwF possesses univariate margins belonging to univariate Tweedie EDMs, and it establishes these marginal distributions explicitly. The multivariate Tweedie family of distributions can now be naturally defined.

Definition 3.1. The joint distribution of $\mathbf{X}=A \mathbf{Y}$, denoted by $\mathbf{X} \sim T w_{n, p}(\boldsymbol{\theta}, \tilde{\lambda})$, is the $n$-variate additive $e^{3}$ Tweedie distribution. Here, $\boldsymbol{\theta} \in \mathbf{\Theta} \subset \mathbf{R}^{n}$ is the $n$-variate vector of canonical parameters where $\mathbf{\Theta}$ is the Cartesian product of the domains $\Theta \subset \mathbf{R}$.

It can be shown that MTwF is obtained as a solution of a generalized Cauchy's functional equation. More precisely, given the aforementioned setup, every univariate margin $X_{j}$ of the $n$-variate random vector $\mathbf{X}$ is an EDM if and only if the corresponding function $\kappa(x)$ is the solution of the following functional equation:

$$
\kappa(x y)=\kappa(x) f(y)+g(y) .
$$

In view of the above, the multivariate Tweedie family is the only possible multivariate extension of EDMs, given matrix (3.3) and Definition 3.1.

3 Tweedie EDMs can be formulated in both the additive and reproductive forms. Thus, discussing one form only does not imply any restrictions on the produced results. 


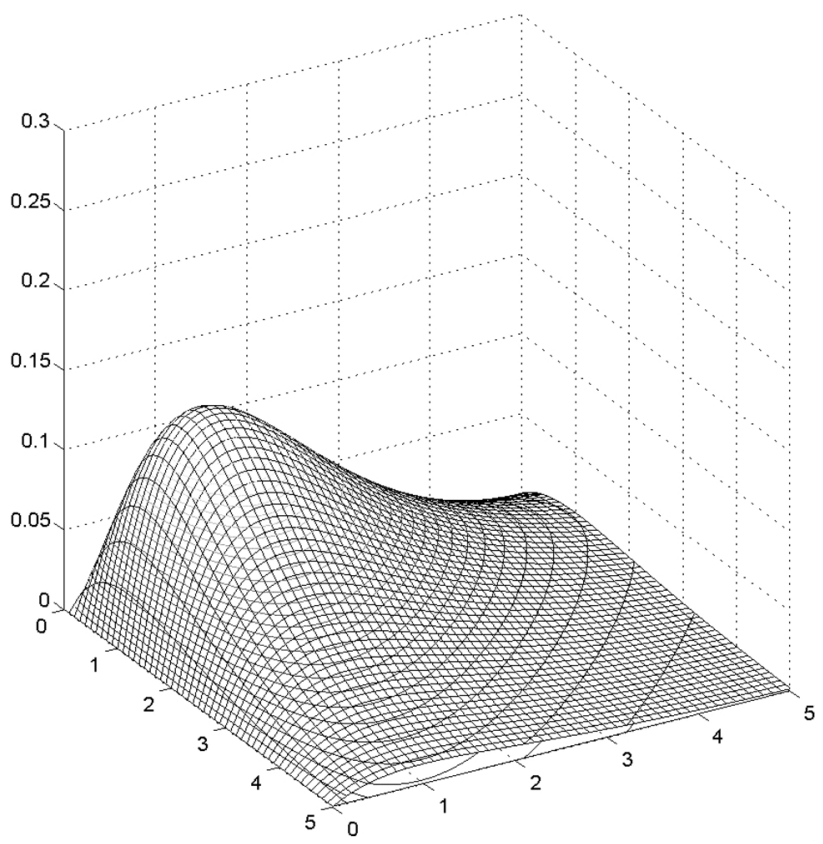

FIGURE 3.1: Bivariate gamma with independent univariate marginal distributions.

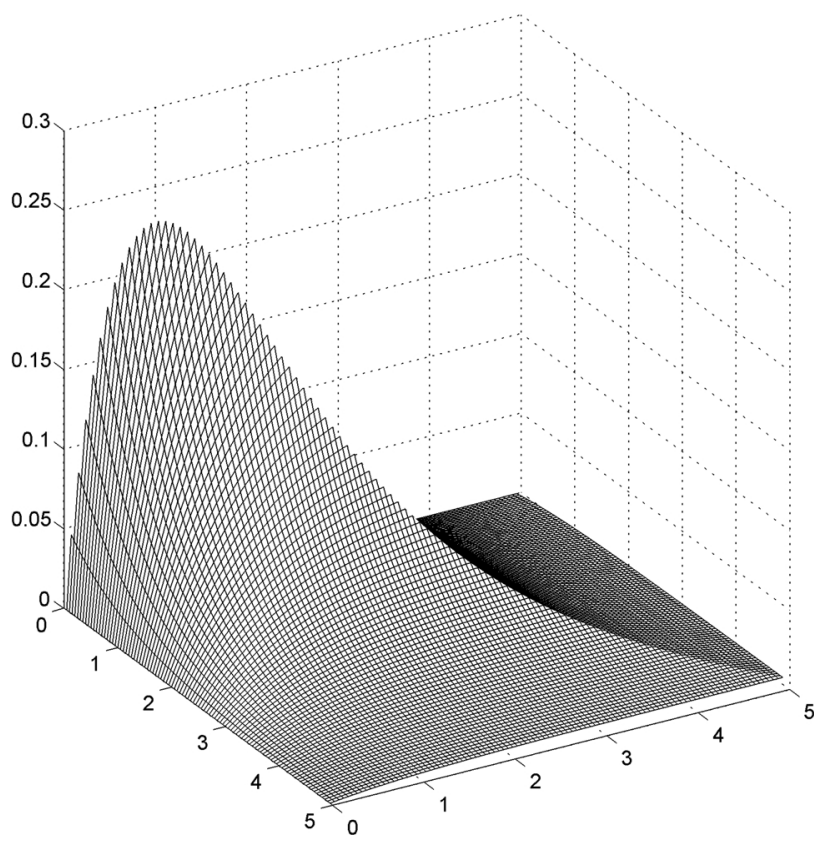

FIGURE 3.2: Bivariate gamma with dependent univariate marginal distributions. 


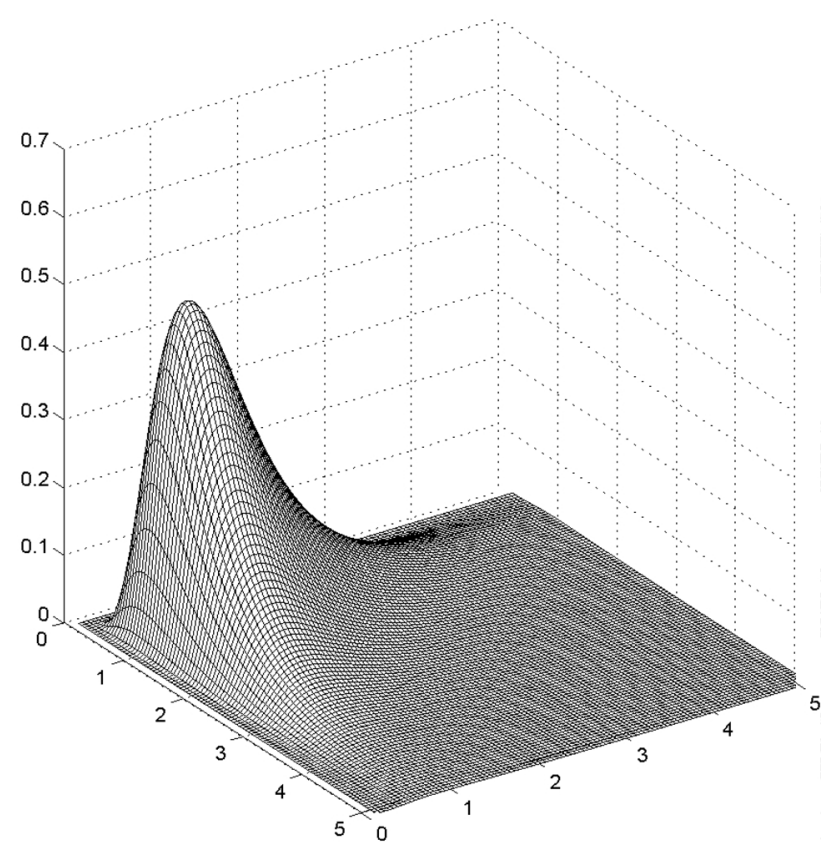

FIgURE 3.3: Bivariate inverse Gaussian with independent univariate marginal distributions.

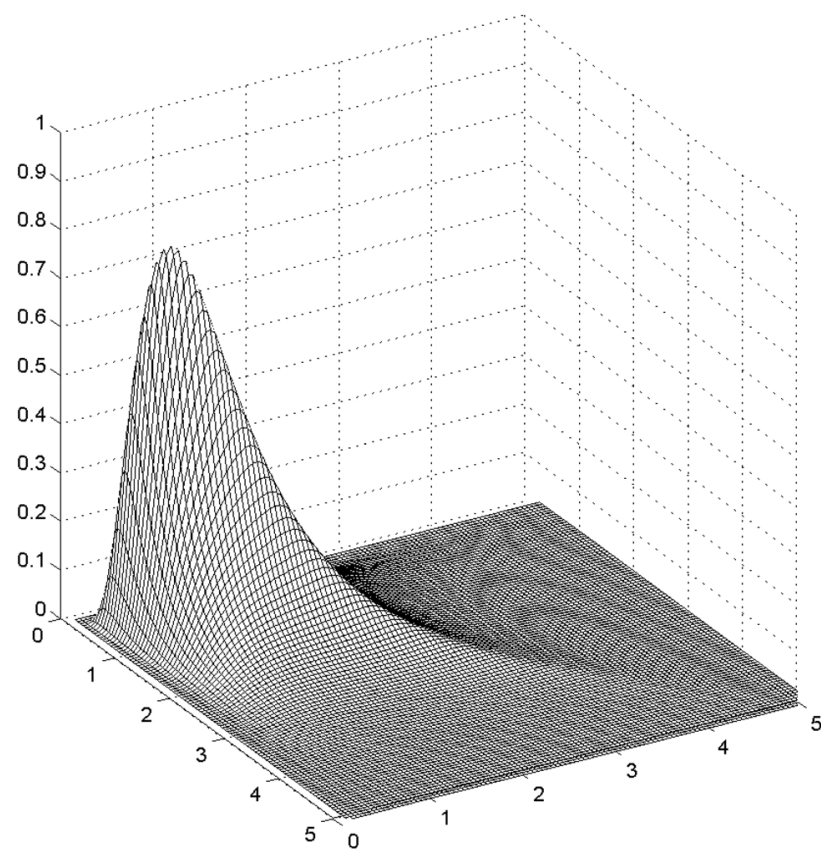

FIgURE 3.4: Bivariate inverse Gaussian with dependent univariate marginal distributions. 
Choosing an appropriate $p$ parameter, we instantly arrive at, say, the multivariate Poisson $(p=1)$, multivariate gamma $(p=2)$, multivariate inverse Gaussian $(p=3)$, and multivariate compound Poisson $(p \in(1,2))$ distributions. It should be emphasized that even more flexibility in modeling (insurance) risks can be obtained by considering multivariate Tweedie distributions corresponding to the non-integer $p$ parameters.

Figures 3.1, 3.2, 3.3 and 3.4 compare some bivariate members of $\mathrm{MTwF}$ with independent and dependent univariate marginal distributions. It can be clearly observed that in the framework of the proposed family dependent risks are riskier than the independent ones.

\subsection{The multivariate Tweedie compound Poisson distribution}

We now consider $T w_{n, p}(\boldsymbol{\theta}, \tilde{\lambda})$ when $p$ is in the interval $(1,2)$ (in what follows, it is denoted by $T w_{n,(1,2)}(\boldsymbol{\theta}, \tilde{\lambda})$. This case relates to the multivariate compound Poisson distributions with gamma severities, and is further referred to as the multivariate Tweedie compound Poisson distribution. It is important in, say, the insurance industry due to the fact that it allows for randomness both in claims' frequencies and amounts.

Let $N_{0}, N_{1}, \ldots, N_{n}$ denote a sequence of mutually independent Poisson rv's, such that, for $i=0,1, \ldots, n, N_{i} \sim \operatorname{Poisson}\left(\lambda_{i} \kappa_{p}(\theta)\right)$. Also, for $k=0,1, \ldots$, let $Y_{i, k} \backsim G a(\theta,-\alpha)$, be mutually independent and identically distributed gamma rv's independent of $N_{i}$, where $\theta, \alpha<0$ and $\alpha=(p-2) /(p-1)$.

Denote by

$$
Y_{i}=\left\{\begin{array}{ll}
\sum_{k=1}^{N_{i}} Y_{i, k}, & N_{i}>0 \\
0, & N_{i}=0
\end{array},\right.
$$

the Tweedie compound Poisson rv, which can also be written as $Y_{i} \backsim T w_{(1,2)}\left(\theta, \lambda_{i}\right)$. Then, the pdf of $Y_{i}$ can be formulated as

$$
f_{Y_{i}}(y)=h\left(y ; \lambda_{i}\right) \exp \left(\theta y-\lambda_{i} \kappa_{p}(\theta)\right),
$$

with

$$
h(y ; \lambda)=\sum_{n=1}^{\infty} \frac{\left(\lambda \kappa_{p}\left(-\frac{1}{y}\right)\right)^{n}}{y \Gamma(-n \alpha) n !}
$$

for $y>0$, and

$$
f_{Y_{i}}(0)=P\left(Y_{i}=0\right)=P(N=0)=\exp \left(-\lambda_{i} \kappa_{p}(\theta)\right),
$$

otherwise. 
We now have the required random vector $\mathbf{Y}=\left(Y_{0}, Y_{1}, \ldots, Y_{n}\right)^{T}$ and, considering common $\theta$ parameters and applying Definition 3.1, we arrive at the resulting multivariate compound Poisson distribution, that is $\mathbf{X} \sim T w_{n,(1,2)}(\boldsymbol{\theta}, \tilde{\lambda})$, where $\boldsymbol{\theta}$ is a vector of $\theta$ 's and $\tilde{\lambda}=\left(\lambda_{0}+\lambda_{1}, \lambda_{0}+\lambda_{2}, \ldots, \lambda_{0}+\lambda_{n}\right)^{T}$ (cf. Theorem 3.1).

Also, we have that the aggregate risk is given by

$$
S=\sum_{j=1}^{n} X_{j}=n \sum_{k=1}^{N_{0}} Y_{0, k}+\sum_{j=1}^{n} \sum_{k=1}^{N_{j}} Y_{j, k},
$$

and thus, the distribution of $S$ is not a Tweedie compound Poisson. Indeed, 1) after setting $\theta_{0} / \theta_{j}=n$ and $\lambda_{j}=0$ in Theorem 3.1, we obtain that

$$
n Y_{0}=n \sum_{k=1}^{N_{0}} Y_{0, k} \sim T w_{(1,2)}\left(\theta / n, \lambda_{0} n^{\alpha}\right)
$$

and 2) the distribution of the double sum in equation (3.4) is

$$
\sum_{k=1}^{N_{j}} Y_{j, k}=\sum_{j=1}^{n} \sum_{k=1}^{N_{j}} Y_{j, k} \backsim T w_{(1,2)}(\theta, \lambda),
$$

where $\lambda=\lambda_{1}+\lambda_{2}+\cdots+\lambda_{n}$. In light of this, the distribution of $S$ is a compound Poisson with Poisson parameter $\Lambda=\lambda_{0} n^{\alpha}+\lambda$ and the corresponding severity distribution possessing the cdf

$$
F(s)=\frac{1}{\Lambda}\left(\lambda_{0} n^{\alpha} G(s ; \theta / n,-\alpha)+\lambda G(s ; \theta,-\alpha)\right) .
$$

We conclude this section with other useful properties of Tweedie compound Poisson rv's:

- The expectation of $X_{j}$ is $\mathbf{E}\left[X_{j}\right]=\left(\lambda_{0}+\lambda_{j}\right) \kappa_{p}(\theta) \alpha \theta^{-1}$.

- The variance of $X_{j}$ is $\operatorname{Var}\left[X_{j}\right]=\left(\lambda_{0}+\lambda_{j}\right) \kappa_{p}(\theta)(\alpha-1) \alpha \theta^{-2}$.

- The correlation between $X_{i}$ and $X_{j}, i \neq j$ is $\operatorname{Corr}\left[X_{i}, X_{j}\right]=\frac{\lambda_{0}}{\sqrt{\left(\lambda_{0}+\lambda_{j}\right)\left(\lambda_{0}+\lambda_{i}\right)}}$.

\section{TCE AND THE RELATED CAPITAL-AT-RISK DECOMPOSITION RULE}

We have so far considered two of the three pillars of the so-called advanced risk measurement, i.e., the multivariate probabilistic model and the risk functional to measure the degree of riskiness it implies (cf., e.g., Denuit et al., 2005 for the definition of 'riskiness'). In the two following theorems we evaluate equations (1.1) and (1.3) in the general framework of MTwF. Namely, we first 
derive the economic capital required for the overall risk $S$, and we establish the amount of capital to be set aside due to its part $X$, thereafter.

Recall that we denote by $S=X_{1}+X_{2}+\cdots+X_{n}$ the aggregate risk of the financial conglomerate, which, according to Definition 3.1, is generally written as

$$
S=\eta Y_{0}+\sum_{j=1}^{n} Y_{j}
$$

subject to $\eta=\sum_{j=1}^{n} \frac{\theta_{0}}{\theta_{j}}$.

Theorem 4.1. Let $\mathbf{X} \backsim T w_{n, p}(\boldsymbol{\theta}, \tilde{\lambda})$ be an $M T w F$ random vector. The tail conditional expectation risk measure for $S$ is then formulated as

$T C E_{q}[S]=\eta \mathbf{E}\left[Y_{0}\right] \frac{\bar{F}_{S-\eta Y_{0}+\left(\eta Y_{0}\right)^{*}}\left(\operatorname{VaR}_{q}[S]\right)}{\bar{F}\left(\operatorname{VaR}_{q}[S]\right)}+\mathbf{E}[Y] \frac{\bar{F}_{S-Y+Y^{*}}\left(\operatorname{VaR}_{q}[S]\right)}{\bar{F}\left(\operatorname{VaR}_{q}[S]\right)}$,

where $Y=\sum_{j=1}^{n} Y_{j}$, and $Y^{*}$ denotes the associated counterpart of $Y$.

Proof. Follows from the additive property of the expectation operator and Lemma 2.1.

Theorem 4.2. Let $\mathbf{X} \sim T w_{n, p}(\boldsymbol{\theta}, \tilde{\lambda})$ be an $M T w F$ random vector. Then the economic capital allocation based on TCE is

$T C E_{q}\left[X_{j} \mid S\right]=\frac{\theta_{0}}{\theta_{j}} \mathbf{E}\left[Y_{0}\right] \frac{\bar{F}_{S-\eta Y_{0}+\left(\eta Y_{0}\right)^{*}}\left(\operatorname{VaR}_{q}[S]\right)}{\bar{F}\left(\operatorname{VaR}_{q}[S]\right)}+\mathbf{E}\left[Y_{j}\right] \frac{\bar{F}_{S-Y_{j}+Y_{j}^{*}}\left(\operatorname{VaR}_{q}[S]\right)}{\bar{F}\left(\operatorname{VaR}_{q}[S]\right)}$

Proof. First note that

$$
T C E_{q}\left[X_{j} \mid S\right]=\frac{\theta_{0}}{\theta_{j}} \mathbf{E}\left[Y_{0} \mid S>\operatorname{VaR}_{q}[S]\right]+\mathbf{E}\left[Y_{j} \mid S>\operatorname{VaR}_{q}[S]\right]
$$

according to the additive property of conditional expectations.

Although the expression for the first term of the right hand side of the above equation does not follow from Lemma 2.1 directly, it can be derived in a similar fashion, i.e.,

$$
\begin{aligned}
\mathbf{E}\left[Y_{0} \mid S>\operatorname{VaR}_{q}[S]\right] & =\mathbf{E}\left[Y_{0}\right]-\mathbf{E}\left[Y_{0} \mathbf{1}\left(S \leq \operatorname{VaR}_{q}[S]\right)\right] \\
& =\frac{\mathbf{E}\left[Y_{0}\right]-\int_{0}^{\operatorname{VaR}_{q}[S] / \eta} u f_{Y_{0}}(u) F_{S-\eta Y_{0}}\left(\operatorname{VaR}_{q}[S]-\eta u\right) d u}{\bar{F}\left(\operatorname{VaR}_{q}[S]\right)},
\end{aligned}
$$


which after the change of variables $t=\eta u$ and noticing that $f_{Y_{0}}(u)=\eta f_{\eta Y_{0}}(t)$, yields

$$
\begin{aligned}
\mathbf{E}\left[Y_{0} \mid S>\operatorname{VaR}_{q}[S]\right]=\frac{\mathbf{E}\left[Y_{0}\right]-\int_{0}^{\operatorname{VaR} R_{q}[S]} \frac{1}{\eta} t f_{\eta Y_{0}}(t) F_{S-\eta Y_{0}}\left(\operatorname{VaR}_{q}[S]-t\right) d t}{\bar{F}\left(\operatorname{VaR}_{q}[S]\right)} \\
=\frac{\mathbf{E}\left[Y_{0}\right]-\mathbf{E}\left[\eta Y_{0}\right] \int_{0}^{\operatorname{VaR} R_{q}[S]} \frac{1}{\eta} f_{\left(\eta Y_{0}\right)^{*}}(t) F_{S-\eta Y_{0}}\left(\operatorname{VaR}_{q}[S]-t\right) d t}{\bar{F}\left(\operatorname{VaR}_{q}[S]\right)} \\
=\frac{\mathbf{E}\left[Y_{0}\right]\left(1-\int_{0}^{\operatorname{VaR} q}[S] F_{S-\eta Y_{0}}\left(\operatorname{VaR}_{q}[S]-t\right) d F_{\left(\eta Y_{0}\right)^{*}}(t)\right)}{\bar{F}\left(\operatorname{VaR}_{q}[S]\right)} \\
=\mathbf{E}\left[Y_{0}\right] \frac{\bar{F}_{S-\eta Y_{0}+\left(\eta Y_{0}\right)^{*}}\left(\operatorname{VaR}_{q}[S]\right)}{\bar{F}\left(\operatorname{VaR}_{q}[S]\right)} .
\end{aligned}
$$

The expression for $\mathbf{E}\left[Y_{j} \mid S>\operatorname{VaR}_{q}[S]\right]$ follows directly from Lemma 2.1 and can be formulated as

$$
\mathbf{E}\left[Y_{j} \mid S>\operatorname{VaR}_{q}[S]\right]=\mathbf{E}\left[Y_{j}\right] \frac{\bar{F}_{S-Y_{j}+Y_{j}^{*}}\left(\operatorname{VaR}_{q}[S]\right)}{\bar{F}\left(\operatorname{VaR}_{q}[S]\right)},
$$

which completes the proof.

In what follows we shall illustrate Theorems 4.1 and 4.2 by an example where the underlying random vector follows Tweedie compound Poisson distributions.

\section{ECONOMIC CAPITALS AND MULTIVARIATE TWEEDIE COMPOUND POISSON DISTRIBUTIONS}

In the previous section and in Section 2, we pointed out that closure under the 'associated transform' essentially simplifies calculations of TCE and the economic capital decomposition rule based on it. We further show that, although the aforementioned closure does not hold in the context of Tweedie compound Poisson rv's, a convenient representation of the associated counterpart can still be found.

Let $S \sim T w_{(1,2)}(\theta, \lambda)$ be the univariate Tweedie compound Poisson rv. We establish the second part of the above statement as Lemma 5.1, while the first part follows from

$$
f_{S^{*}}(s)=\frac{s f(s)}{\mathbf{E}[S]}=\exp \left(\theta s-\lambda \kappa_{p}(\theta)\right) \sum_{k=1}^{\infty} \frac{(-\theta s)^{-\kappa \alpha+1}}{s \Gamma(-k \alpha+1)} \frac{\left(\lambda \kappa_{p}(\theta)\right)^{k-1}}{(k-1) !},
$$


which is of the form

$$
h(s ; \lambda, \theta) \exp \left(\theta x-\lambda \kappa_{p}(\theta)\right)
$$

and therefore $S^{*}$ is not a Tweedie compound Poisson. In fact, it is not an EDM either, since $h(\cdot)$ is dependent on $\theta$.

Lemma 5.1. Let $S \backsim T w_{(1,2)}(\theta, \lambda)$ and $S^{*}$ be its associated counterpart. Then $S^{*} \stackrel{d}{=}$ $S+\xi$, where $\xi \sim T w_{2}(\theta,-\alpha-1)$.

Proof. Let $t w_{2}(\cdot ; \theta,-\alpha)$ denote the pdf of Tweedie rv with $p=2$ (gamma distribution), and $N$ be Poisson rv having pdf $t w_{1}(\cdot ; \lambda)$. Then, we can rewrite the density of $S^{*}$ as

$$
\begin{aligned}
f_{S^{*}}(s) & =\sum_{k=1}^{\infty} e^{-\lambda \kappa_{p}(\theta)} \frac{\left(\lambda \kappa_{p}(\theta)\right)^{k-1}}{(k-1) !} e^{\theta s} \frac{s^{-k \alpha}(-\theta)^{-k \alpha+1}}{\Gamma(-k \alpha+1)} \\
& =\sum_{k=1}^{\infty} t w_{1}\left(k-1 ; \lambda \kappa_{p}(\theta)\right) \cdot t w_{2}(s ; \theta,-k \alpha+1) \\
& =\sum_{k=1}^{\infty} t w_{1}\left(k-1 ; \lambda \kappa_{p}(\theta)\right) \cdot\left(t w_{2}(s ; \theta,-(k-1) \alpha) \circ t w_{2}(s ; \theta,-\alpha+1)\right)
\end{aligned}
$$

where ${ }^{\circ}$ stands for convolution.

Further, conditioning on $N+1=k$, we arrive at

$$
f_{S^{*}}(s)=\sum_{k=1}^{\infty} f_{N+1}(k) \cdot f_{(S+\xi) \mid N+1=k}(s)=f_{S+\xi}(s),
$$

which completes the proof.

We can now formulate the TCE risk measure of $S$ as follows

Theorem 5.1. Let $S \sim T w_{(1,2)}(\theta, \lambda)$, then we have that

$$
T C E_{q}[S]=\lambda \kappa_{p}(\theta) \frac{\alpha}{\theta} \frac{\bar{F}_{S+\xi}\left(\operatorname{VaR}_{q}[S]\right)}{\bar{F}\left(\operatorname{VaR}_{q}[S]\right)} .
$$

Proof. Follows from Lemmas 2.1 and 5.1.

Another way to prove Theorem 5.1 is by the law of total (iterated) expectations. Namely, we obtain that

$$
\operatorname{TCE}_{q}[S]=\mathbf{E}\left[S \mid S>\operatorname{VaR}_{q}[S]\right]=\mathbf{E}\left[\mathbf{E}\left[S\left|S>\operatorname{VaR}_{q}[S]\right| N\right]\right] .
$$


Then the right-most side of the equation above is written as

$$
\mathbf{E}\left[\mathbf{E}\left[S\left|S>\operatorname{VaR}_{q}[S]\right| N=n\right]\right]=\frac{n \alpha}{\theta} \frac{\bar{G}\left(\operatorname{VaR}_{q}[S] ;-n \alpha+1, \theta\right)}{1-q},
$$

and consequently

$$
\begin{aligned}
T C E_{q}[S] & =\frac{1}{1-q} \sum_{k=0}^{\infty} \frac{k \alpha}{\theta} \bar{G}\left(\operatorname{VaR}_{q}[S] ;-k \alpha+1, \theta\right) e^{-\lambda \kappa_{p}(\theta)} \frac{\left(\lambda \kappa_{p}(\theta)\right)^{k}}{k !} \\
& =\frac{\lambda \kappa_{p}(\theta)}{1-q} \frac{\alpha}{\theta} \int_{\operatorname{VaR}_{q}[S]}^{\infty} \sum_{k=0}^{\infty} e^{\theta x} \frac{x^{-k \alpha}(-\theta)^{-k \alpha+1}}{\Gamma(-k \alpha+1)} e^{-\lambda \kappa_{p}(\theta)} \frac{\left(\lambda \kappa_{p}(\theta)\right)^{k-1}}{(k-1) !} d x \\
& =\frac{\alpha \lambda \kappa_{p}(\theta)}{\theta} \frac{\bar{F}_{S^{*}}\left(\operatorname{VaR}_{q}[S]\right)}{1-q}=\frac{\alpha \lambda \kappa_{p}(\theta)}{\theta} \frac{\bar{F}_{S+\xi}\left(\operatorname{VaR}_{q}[S]\right)}{1-q},
\end{aligned}
$$

which confirms Theorem 5.1.

We further consider multivariate dependent Tweedie compound Poisson distributions.

Corollary 5.1. Let $\mathbf{X} \sim T w_{n,(1,2)}(\boldsymbol{\theta}, \tilde{\lambda})$ be a Tweedie compound Poisson random vector, and $S$ be the sum of its univariate marginal constituents. Then the tail conditional expectation risk measure and the economic capital allocation derived from it are formulated correspondingly as

$T C E_{q}[S]=\frac{n \lambda_{0} \kappa_{(1,2)}(\theta) \alpha}{\theta} \frac{\bar{F}_{S+n \xi}\left(\operatorname{VaR}_{q}[S]\right)}{\bar{F}\left(\operatorname{VaR}_{q}[S]\right)}+\frac{\lambda \kappa_{(1,2)}(\theta) \alpha}{\theta} \frac{\bar{F}_{S+\xi}\left(\operatorname{VaR}_{q}[S]\right)}{\bar{F}\left(\operatorname{VaR}_{q}[S]\right)}$

and

$T C E_{q}\left[X_{j} \mid S\right]=\frac{\lambda_{0} \kappa_{(1,2)}(\theta) \alpha}{\theta} \frac{\bar{F}_{S+n \xi}\left(\operatorname{VaR}_{q}[S]\right)}{\bar{F}\left(\operatorname{VaR}_{q}[S]\right)}+\frac{\lambda_{j} \kappa_{(1,2)}(\theta) \alpha}{\theta} \frac{\bar{F}_{S+\xi}\left(\operatorname{VaR}_{q}[S]\right)}{\bar{F}\left(\operatorname{VaR}_{q}[S]\right)}$,

where $\lambda=\lambda_{1}+\lambda_{2}+\cdots+\lambda_{n}$ and $\xi \sim T w_{2}(\theta,-\alpha-1)$.

Proof. Follows from Theorems 4.1 and 4.2 and Lemma 5.1.

We also note that in this case, the additivity of the allocation rule can be easily observed from the two latter formulas. 


\section{Concluding COMMENTS}

Multivariate dependent probabilistic models having convenient dependence structures, suitable risk functionals, and the ability to evaluate the latter in the framework of the former are three main pillars of the successful risk measurement, which is a natural precursor of the even more demanding process of managing financial risks.

It must be emphasized that every one of the above mentioned concepts bears its own specific challenge making the whole process far from straightforward. For instance, there generally exist a considerable number of methods of multivariate modeling univariate margins which are followed by adding a dependence structure. However, the insurance and finance industries dictate specific laws that must be obeyed. Namely, mostly only multivariate models defined on $\mathbf{R}_{+}^{n}$, preserving unimodality and positive skewness, can serve as appropriate candidates for model insurance losses. These peculiarities discard, for instance, the elliptical family of multivariate distributions, although it is quite useful in general finance. Also, there is a trade-off between, on the one hand, the approximation level provided by the model and on the other, its analytic complexity. The present popular copula multivariate structures, for example, lead to some essential analytic complications in both inference and risk measurement. Consequently, one has to impose an additional restriction of tractability on the choice of the multivariate cdf and its dependence structure. This often leads to rejecting more complicated models, although they might have described insurance losses well.

The choice of a suitable risk measure is not obvious either. As it has been already stressed, a significant number of risk measures of various kinds exist nowadays, the earliest of which would seem to be the Value-at-Risk and the latest - the distorted risk measures, the measures based on the tail variance and weighted pcp's. Several axiomatic approaches to risk measurement have also been developed. The debate over what risk measure to apply is still far from being over.

Needless to say, the necessary evaluation of the chosen risk measure in the context of the multivariate probability distribution describing risks is also rather challenging. Indeed, abandoning, say, the independence assumption makes the model more realistic but at the same time much less tractable analytically. The advanced nature of today's most popular risk measures along with the formally unpleasant probability distribution functions of the models also lead to a highly challenging problem.

In this paper we have made an attempt to resolve the complications above, when the underlying probabilistic model is the multivariate Tweedie family proposed herein, and the risk measure is the well-known tail conditional expectation. Moreover, the more complicated issue of the consequent allocation of the economic capital to its various constituents has also been considered in the general framework of the aforementioned set-up. 


\section{REFERENCES}

ACERBI, C. and TASChE, D. (2002) On the coherence of expected shortfall. Journal of Banking \& Finance 26(7), 1487-1503.

Artzner, P., Delbaen, F., Eber, J.M. and Heath, D. (1999) Coherent measures of risk. Mathematical Finance 9, 203-228.

Bildikar, S. and Patil, G.P. (1968) Multivariate exponential type distributions. Annals of Statistics 39(4), 1316-1326.

Brazauskas, V., Jones, B.L., Puri, M.L. and Zitikis, R. (2008) Estimating Conditional Tail Expectation with Actuarial Applications in View. Journal of Statistical Planning and Inference 138(11), 3590-3604.

CAI, J. and LI, H. (2005) Conditional tail expectations for multivariate phase-type distributions. Journal of Applied Probability 42(3), 810- 825.

Chiragiev, A. and Landsman, Z. (2007) Multivariate Pareto portfolios: TCE-based capital allocation and divided differences. Scandinavian Actuarial Journal 4, 261-280.

Denault, M. (2001) Coherent allocation of risk capital. Journal of Risk 4(1), 7-21.

DenNeberG, D. (1994) Non-additive measure and integral. Kluwer, Dordrecht.

Denuit, M., Dhaene, J., Goovaerts, M. and KaAs, R. (2005) Actuarial theory for dependent risks - measures, orders and models. John Wiley \& Sons.

Dhaene, J., Henrard, L., Landsman, Z., Vandendorpe, A. and Vanduffel, S. (2008) Some results on the CTE-based capital allocation rule. Insurance: Mathematics and Economics, (in press).

Dhaene, J., Vanduffel, S., Goovaerts, M., KaAs, R., Tang, Q. and Vyncke, D. (2006) Risk measures and comonotonicity: a review. Stochastic Models 22, 573-606.

Furman, E. (2008) On a multivariate Gamma distribution. Statistics and Probability Letters, 78(15), 2353-2360.

Furman, E. and LANDSMAN, Z. (2005) Risk capital decomposition for a multivariate dependent gamma portfolio. Insurance: Mathematics and Economics 37(3), 635-649.

FURMAN, E. and LANDSMAN, Z. (2006a) On some risk-adjusted tail-based premium calculation principles. Journal of Actuarial Practice 13, 175-191.

Furman, E. and LANDSMAN, Z. (2006b) Tail variance premium with applications for elliptical portfolio of risks. ASTIN Bulletin 36(2), 433-462.

FuRMAN, E. and ZiTIKIS, R. (2008a) Weighted premium calculation principles, Insurance: Mathematics and Economics 42(1), 459-465.

Furman, E. and Zitikis, R. (2008b) Weighted risk capital allocations. Insurance: Mathematics and Economics 43(2), 263-269.

Furman, E. and ZiTIKIS, R. (2008c) General Stein-type decompositions of covariances: revisiting the Capital Asset Pricing Model. Available at SSRN: http://ssrn.com/abstract=1103333

HürlimanN, W. (2003) Conditional Value-at-Risk bounds for compound Poison risks and a normal approximation. Journal of Applied Mathematics 3, 141-153.

HÜRLIMANN, W. (2004) Multivariate Fréchet copulas and conditional value-at-risk. International Journal of Mathematics and Mathematical Sciences 7, 345-364.

HürLimanN, W. (2006) A note on generalized distortion risk measures. Finance Research Letters 3(4), 267-272.

HÜrlimann, W. (2007a) Positive dependence properties of the multivariate reduction class. Far East Journal of Theoretical Statistics 21(2), 157-169.

JoE, H. (1997) Multivariate models and dependence concepts. Chapman \& Hall, London.

JoRgensen, B. (1997) The theory of dispersion models. Chapman \& Hall, London.

KaAs, R., Goovaerts, M.J., Dhaene, J. and Denuit, M. (2001). Modern actuarial risk theory. Kluwer Academic Publishers.

Landsman, Z. and Valdez, E. (2003) Tail conditional expectation for elliptical distributions. North American Actuarial Journal 7(4), 55-71.

Landsman, Z. and ValdeZ, E. (2005) Tail conditional expectations for exponential dispersion models. ASTIN Bulletin 35(1), 189-209.

Leavens, D.H. (1945) Diversification of investments. Trusts and Estates 80, 469-473.

MARDiA, K.V. (1970) Families of bivariate distributions, Griffin, London. 
McNeil, A.J., Frey, R. and Embrechts, P. (2005) Quantitative risk management, Princeton University Press, Princeton and Oxford.

PANJer, H.H. and JiA, J. (2001) Solvency and capital allocation. Institute of Insurance and Pension Research, University of Waterloo, Research Report N 01-14.

PATIL, G.P. and ORD, J.K. (1976) On size-biased sampling and related form-invariant weighted distributions, Sankhya, Ser. B 38, 48-61.

PatiL, G.P. and RAO, C.R. (1978) Weighted distributions and size-biased sampling with applications to wildlife populations and human families. Biometrics 34, 179-189.

TASCHE, D. (2002) Expected shortfall and beyond. Journal of Banking and Finance 26(7), 15191533.

VERNIC, R. (2006) Multivariate skew-normal distributions with applications in insurance. Insurance: Mathematics \& Economics 38, 413-426.

WANG, S.S. (1995) nsurance pricing and increased limits ratemaking by proportional hazards transforms. Insurance: Mathematics and Economics 17, 43-54.

WANG, S.S. (1996) Premium calculation by transforming the layer premium density. ASTIN Bulletin 26, 71-92.

WANG, S., Young, V.R. and PANJER, H.H. (1997) Axiomatic characterization of Insurance prices. Insurance: Mathematics and Economics 21, 173-183.

Zaik, E., WAlter, J., Kelling, G. and JAmes, C. (1996) RAROC at bank of America: from theory to practice. Journal of Applied Corporate Finance 9(2), 83-93.

\section{EDWARD FURMAN}

Department of Mathematics and Statistics

York University

Toronto, $\mathrm{ON}$

Canada, M3J 1 P3

E-mail:efurman@mathstat.yorku.ca

ZiNOVIY LANDSMAN

Department of Statistics

University of Haifa

Haifa, Mount Carmel 31905

Israel

E-mail: landsman@stat.haifa.ac.il 